\title{
CARACTERIZAÇÃO DE FILMES ORODISPERSÍVEIS FORMULADOS COM FLAVORIZANTES NATURAIS
}

\section{ARTIGO ORIGINAL}

TESCAROLLO, lara Lúcia ${ }^{1}$

OLIVEIRA, Aratã Carvalho ${ }^{2}$

FIORIN, Arielli Pereira Couro ${ }^{3}$

NASCIMENTO, Mariana Ferreira ${ }^{4}$

TESCAROLLO, lara Lúcia. Et al. Caracterização de filmes orodispersíveis formulados com flavorizantes naturais. Revista Científica Multidisciplinar Núcleo do Conhecimento. Ano 04, Ed. 10, Vol. 06, pp. 05-17. Outubro de 2019. ISSN: 24480959, Link de acesso: https://www.nucleodoconhecimento.com.br/saude/filmesorodispersiveis

\section{RESUMO}

A via oral é uma das mais utilizadas para a administração de formas farmacêuticas sólidas por ser conveniente, econômica e de fácil administração, entretanto, pode

1 Professora da Universidade São Francisco, Doutorado em Fármacos e Medicamentos; Mestrado em Fármacos e Medicamentos; Especialização em Ciências Biológicas; Graduação em Ciências Farmacêuticas. Membro do Grupo de Pesquisa em Meio Ambiente e Sustentabilidade.

2 Acadêmica de Iniciação Científica da Universidade São francisco, Curso de Farmácia.

3 Acadêmica de Iniciação Científica da Universidade São francisco, Curso de Farmácia.

4 Acadêmica de Iniciação Científica da Universidade São francisco, Curso de Farmácia. 
apresentar como limitações a dificuldade de deglutição para alguns pacientes. Os filmes orodispersíveis (FODs) se constituem numa forma farmacêutica, inovadora, prática, versátil e de fácil administração. São caracterizados por filmes poliméricos, finos, flexíveis desenvolvidos para administração de fármacos por via oral e podem superar as dificuldades de deglutição associadas às formas farmacêuticas sólidas como comprimidos e cápsulas. Além do fármaco, formas farmacêuticas orodispersíveis têm em sua composição polímeros formadores de filme, plastificantes, edulcorantes, agente estimulante da saliva, aromatizantes, corantes, estabilizadores, espessantes, potencializadores da permeação e desintegrantes. Os edulcorantes, aromatizantes e flavorizantes conferem aos FODs um sabor adocicado e odor agradável e podem contribuir no mascaramento de fármacos excessivamente amargos. O presente estudo teve como objetivo desenvolver e avaliar as propriedades físico-químicas e organolépticas de FODs formulados com dois diferentes flavorizantes a fim de que possam ser utilizados para veicular fármacos com potencialidade de absorção via oral. Foram produzidas duas amostras empregandose os óleos essências de limão siciliano e tangerina. As amostras foram submetidas ao teste preliminar de estabilidade e avaliadas quanto ao aspecto, cor, odor, sabor, $\mathrm{pH}$, tempo de desintegração e peso médio. Os resultados revelaram potencialidade no uso de óleos essências de limão siciliano e tangerina no desenvolvimento de FODs, entretanto, no estudo de estabilidade preliminar foram observadas alterações muito perceptíveis para as amostras armazenadas em diferentes condições de temperatura. Embora outros estudos mais aprofundados devam ser conduzidos na caraterização das amostras produzidas, a abordagem adotada neste estudo pode contribuir na construção de uma base científica para desenvolvimento de preparações envolvendo FODs.

Palavras-chave: Biopolímeros, óleos essenciais, tecnologia farmacêutica.

\section{INTRODUÇÃO}

A via oral é uma das mais utilizadas para a administração de formas farmacêuticas sólidas por ser conveniente, econômica e de fácil administração, entretanto, pode apresentar como limitações a dificuldade de deglutição para pacientes pediátricos e 
geriátricos. Sistemas orodispersíveis (SODs), foram desenvolvidos para superar as dificuldades de deglutição associadas às formas farmacêuticas sólidas como comprimidos e cápsulas ( GHOSH et al, 2011; BALA et al., 2013). Assim, começaram a ganhar popularidade devido suas vantagens como a rápida desintegração e dissolução, autoadministração, não sendo necessária a mastigação e a ingestão de água (ARYA et al., 2010). Também se dissolvem rapidamente quando colocados sobre a língua (ORLU et al., 2017), permitindo melhor absorção de fármacos (HOFFMANN; BREITENBACH; BREITKREUTZ, 2014). Essa característica em particular, permite maior disponibilidade e eficácia do fármaco (PREIS; PEIN; BREITKREUTZ, 2012).

Um dos primeiros filmes orais foi desenvolvido pela Pfizer, denominado Listerine ${ }^{\circledR T M} \mathrm{e}$ foi usado como antisséptico bucal no combate ao mau hálito. A formulação desse tipo de filme envolve o uso de polímeros formadores de filme, plastificantes, diferentes fármacos, edulcorantes, agente estimulante da saliva, aromatizantes, corantes, estabilizadores, espessantes, potencializadores da permeação e desintegrantes (BALA et al., 2013). Edulcorantes conferem ao filme um sabor adocicado e aromatizantes um odor agradável. Flavorizantes também são empregados para corrigir, ao mesmo tempo, o sabor e odor das formulações bem como melhorar ou mascarar 0 odor e sabor desagradável de alguns fármacos (HOFFMANN; BREITENBACH; BREITKREUTZ, 2014).

Recentemente foram propostas várias formas de dosagem para mucosas, incluindo filmes orodispersíveis (FODs), comprimidos adesivos e géis (PREIS; PEIN; BREITKREUTZ, 2012; BALA et al., 2013). A função promissora dos FODs consiste em melhorar a administração de fármacos com diferentes ações farmacológicas. Estudos apontam que FODs formulados com polímeros naturais e biocompatíveis como goma de caju e goma gelana, tem se mostrado bastante viáveis para administração de insulina, tendo como vantagens a redução dos inconvenientes de degradação enzimática do fármaco no trato digestório e baixa permeabilidade intestinal (RIBEIRO et al., 2017). Outros filmes à base de gelatina pura e a mistura binária entre amido e gelatina se mostraram como alternativas viáveis no 
desenvolvimento de FODs para a liberação de princípios ativos na cavidade bucal (PEREIRA et al., 2019). A incorporação de anestésicos locais como os cloridratos de prilocaína e lidocaína em filmes poliméricos hidrofílicos foi estudada por Couto (2015). Nessa perspectiva os filmes mucoadesivos se apresentaram como alternativa não invasiva na administração de anestésicos bucais com vantagens na redução de custos, submissão do paciente, facilidade de aplicação e menores riscos de contaminação e intoxicações. Outro estudo demonstrou que foi possível formular FODs com cloridrato de diciclomina com a intenção de obter melhor eficiência terapêutica com aumento da biodisponibilidade e melhoria da adesão do paciente. $O$ plastificante usado polietilenoglicol (PEG-400) resultou melhores filmes em relação a parâmetros físico-químicos avaliados. $\mathrm{O}$ aspartame foi usado como edulcorante mascarando o sabor amargo do fármaco (TOMAR et al., 2012). Além da área farmacêutica, o uso de filmes poliméricos dispersíveis tem sido estudado na área cosmética (SANFELICE et al., 2010)

Conforme descrito por Resta e Mali (2019) as formulações de FODs podem ser produzidas pelo processo de casting (evaporação de solvente). Este processo envolve a mistura do polímero em solução com um substrato, seguida da evaporação do solvente, o que proporciona orientação molecular das moléculas do polímero resultando na formação do filme (DENG et al., 2018). Dependendo da espessura e do tamanho do filme formado após a secagem, determina-se a dose do fármaco ou bioativo que pode ser carreada (MUSAZZI et al., 2018)

Existem algumas dificuldades no desenvolvimento de FODs, como exemplo, doses elevadas de fármacos não se incorporam com facilidade nos formadores de película, há várias limitações técnicas como a necessidade de se padronizar a espessura do filme para garantir a uniformidade da dose. A escolha do tipo de embalagem também é importante, pois os FODs são relativamente frágeis exigindo proteção frente à umidade e altas temperaturas para evitar sua desintegração. Para fármacos excessivamente amargos, o mascaramento do sabor desagradável torna-se um grande desafio. Os FODs podem induzir a automedicação (NAND et al., 2010). Os problemas mais comuns dos FODs estão relacionados à sua instabilidade em 
ambientes com alta umidade relativa, a pequena dose de fármaco que pode ser incorporada, essencialmente devido ao seu pequeno tamanho, baixo peso e expessura delgada. Fármacos instáveis no pH bucal ou que podem irritar a mucosa oral não devem ser formulados nesse tipo de sistema (KESHARI; KUMAR SHARMA; PARVEZ, 2014).

O presente estudo teve como objetivo desenvolver e avaliar as propriedades físicoquímicas de FODs formulados com dois diferentes flavorizantes naturais a fim de que possam ser utilizados para veicular fármacos com potencialidade de absorção via oral.

\section{METODOLOGIA}

\subsection{MATERIAL}

Para o desenvolvimento das formulações foram empregadas as diferentes matériasprimas: Goma xantana; Goma carragena, Sorbato de potássio; Benzoato de sódio; Acesulfame k; Sucralose; Lecitina de soja; Manitol; Polissorbato 80; Propilenoglicol; Simeticone 30 \%; Pullulan; Polietilenoglicol 400, água purificada e os óleos essenciais de limão siciliano (Citrus limon) e tangerina (Citrus reticulata).

Os equipamentos utilizados na produção e avaliação das formulações foram: Balança semi-analítica (Marca Gehaka ${ }^{\circledR}$, modelo BG440); pHmetro digital (Marca Gehaka ${ }^{\circledR}$, modelo PG 2000); Laminador (Marca Matrix ${ }^{\circledR}$, modelo BF-FOX MV9102548-6); Placa de vidro (Marca Binder ${ }^{\circledR}$ KBF-115); Estufa (Marca Gehaka ${ }^{\circledR}$, modelo G-4023D); Aquecedor a ar (Marca Mondial ${ }^{\circledR}$, modelo A-08); Guilhotina (Marca Maped Universal ${ }^{\circledR}$, modelo G3208).

\subsection{PREPARO DOS FILMES ORODISPERSÍVEIS}

Os FODs foram produzidos pela técnica casting, em que a solução formadora de filme foi preparada em meio aquoso, colocada sobre um suporte de vidro para desidratação, e após a evaporação do solvente o filme foi removido por destacamento. Foram elaboradas duas formulações variando-se o tipo de flavorizante utilizado. Para o 
desenvolvimento da amostra F1 foram empregadas diferentes matérias-primas como: Goma xantana: 0,05 a 0,07\%; Goma carragena: 0,35 a 0,40\%, Sorbato de potássio: 0,1 a 0,3\%; Benzoato de sódio: 0,05 a 0,15\%; Acesulfame k: 0,2 a 0,5\%; Sucralose: 0,1 a 0,3\%; Lecitina de soja: 0,5 a 1,0\%; Manitol: 0,1 a 0,3\%;, Polissorbato 80: 0,5 a 1,0\%; Propilenoglicol: 1,0 a 2,0\%; Simeticone $30 \%$ : 0,5 a 1,0\%; Pullulan: 15 a 20\%; Polietilenoglicol 400: 1,0 a 2,0\%, Óleo essencial de limão siciliano: 0,5\% e água purificada quantidade suficiente para $100 \%$. Para o desenvolvimento da amostra F2 foram empregados os mesmos componentes variando-se apenas no flavorizante Óleo essencial de tangerina $(0,5 \%)$.

Foram produzidos lotes de bancada de $100 \mathrm{~g}$, com o objetivo de selecionar a melhor formulação. Técnica de preparo da base do filme orodispersível envolveu várias etapas adaptadas a partir de estudos publicados na área (PEREIRA et al., 2019; RESTA e MALI, 2019; COUTO, 2015; FERREIRA et al., 2015).

A produção final envolveu as seguintes etapas: Fase 1: Pesar separadamente a goma xantana, goma carragena, pullulan, sorbato de potássio, benzoato de sódio, acesulfame k, sucralose, manitol e lecitina de soja, dispersar em água destilada, homogeneizar e, em seguida, deixar em repouso até completa dispersão. Fase 2: Pesar separadamente o polissorbato 80 , simeticone emulsão $30 \%$, polietilenoglicol 400, propilenoglicol, homogeneizar, em seguida, deixar em repouso até completa dispersão. Fase 3: Após completa dispersão da Fase 1 e Fase 2, verter uma fase na outra seguida de homogeneização. Fase 4: Acrescentar os flavorizantes. Faze 5: Dispersar a mistura para uma placa de vidro, e distribuir uniformemente com a ajuda do laminador. Fase 6: Secar em estufa com circulação de ar a $30^{\circ} \mathrm{C}$ por 8 horas. Fase 7: Após a secagem, cortar a película com a ajuda da guilhotina no tamanho padronizado. Fase 8: Acondicionar as películas em embalagem apropriada, separadas por um papel manteiga incorporando ao conjunto um sache de sílica para evitar umidade.

Após o preparo dos filmes os mesmos avaliados em relação aos testes de qualidade a fim de se estabelecer as especificações como aspecto, cor, odor, sabor, $\mathrm{pH}$, tempo de desintegração e peso médio. As amostras também foram encaminhadas para o 
estudo preliminar de estabilidade. Os ensaios foram adaptados a partir da Farmacopeia Brasileira (BRASIL, 2019) e estudos desenvolvidos por Sanfelice e colaboradores (2010).

\subsection{CARACTERIZAÇÃO DOS FILMES}

Os filmes foram submetidos a testes de estabilidade preliminar em diferentes condições de armazenamento: estufa a $40^{\circ} \pm 2^{\circ} \mathrm{C}$; geladeira a $5^{\circ} \mathrm{C} \pm 2^{\circ} \mathrm{C}$; temperatura ambiente protegido da luz $\left(25^{\circ} \pm 5^{\circ} \mathrm{C}\right)$ e temperatura ambiente com exposição direta à luz solar $\left(25^{\circ} \pm 5^{\circ} \mathrm{C}\right)$. Os parâmetros avaliados foram: $\mathrm{pH}$; peso-médio; desintegração; sabor, odor e aspecto. As análises foram realizadas nos tempos zero (T0), e a cada 7 dias perfazendo um total de 28 dias. A determinação do aspecto, odor e sabor foi realizada a partir da percepção dos formuladores sendo considerado parâmetro subjetivo.

\subsubsection{ASPECTO}

Cada amostra foi transferida para placa de Petri, observou-se seu aspecto, cor, homogeneidade e uniformidade. $\mathrm{O}$ aspecto foi classificado segundo os seguintes critérios: N (normal, sem alteração visível ou perceptível); LM (levemente modificado); M (modificado); MM (muito modificado).

\subsubsection{ODOR}

Cada amostra foi transferida para placa de Petri, observou-se o odor sendo este caracterizado a partir do tipo de flavorizante utilizado. O odor foi classificado segundo os seguintes critérios: $\mathrm{N}$ (normal, sem alteração, característico do tipo de flavorizante usado); LM (levemente modificado); M (modificado); MM (muito modificado).

\subsubsection{SABOR}

Cada amostra foi transferida para a boca e depositada sob a língua. Após a experiência o sabor foi classificado segundo os seguintes critérios: N (normal, sem 
alteração, característico do tipo de flavorizante usado); LM (levemente modificado); M (modificado); MM (muito modificado).

\subsubsection{DETERMINAÇÃO DO pH}

A determinação do $\mathrm{pH}$ foi realizada utilizando-se potenciômetro acoplado a eletrodo de vidro sensível ao $\mathrm{pH}$. Foram preparadas soluções a $10 \%$. Foram efetuadas três leituras consecutivas em temperatura ambiente.

\subsubsection{TEMPO DE DESINTEGRAÇÃO}

O teste de desintegração foi realizado conforme descrito por Perumal et al. (2008) também referendado por Resta e Mali (2019). Como meio de desintegração foi empregado tampão fosfato salino $(\mathrm{pH} \mathrm{6,8)} \mathrm{para} \mathrm{simular} \mathrm{a} \mathrm{saliva.} \mathrm{Amostras} \mathrm{foram}$ colocadas em $50 \mathrm{~mL}$ de solução tampão fosfato a $37^{\circ} \mathrm{C}$ e mantidas sob agitação. $\mathrm{O}$ tempo de desintegração foi determinado como tempo (segundos) necessário para o filme se desintegrar.

\section{RESULTADOS E DISCUSSÃO}

O desenvolvimento de FODs requer do formulador, um cuidado especial nos atributos relacionados às propriedades organolépticas como o odor e sabor. Conforme descrito por Medeiros e Garruti (2018) a maioria dos fármacos têm intrinsicamente um gosto desagradável e podem provocar reflexos de rejeição inatos nos seres humanos. Um medicamento palatável é aquele em que os atributos sensoriais aversivos foram minimizados, mascarados ou eliminados na sua formulação. Um flavorizante é uma substância natural ou artificial que se adiciona à formulação para dar ou realçar o sabor e o aroma, já o edulcorante confere gosto doce à preparação. $\mathrm{O}$ uso de edulcorantes e flavorizantes é de importância fundamental para melhorar a palatabilidade, pois geralmente ajudam a mascarar o sabor desagradável de um produto. 
A procura de novos sabores é uma das tendências ne desenvolvimento de produtos. Neste estudo foram produzidos dois FODs variando-se apenas no tipo de flavorizante. $\mathrm{Na}$ amostra F1 foi utilizado $0,5 \%$ de óleo essencial de limão siciliano e na F2 0,5\% de óleo essencial de tangerina. Os aromatizantes e flavorizantes são muito importantes na produção de formas farmacêuticas orais, e têm a característica de intensificar não só o aroma como também o sabor. De forma geral, é importante a utilização de corretivos específicos ou empregar técnicas para mascarar o odor e sabor para tornar a preparação palatável e aumentar a adesão do paciente. A escolha do aromatizante ou flavorizante deve estar de acordo com as características do produto (FERREIRA e BRANDÃO, 2008). Neste estudo foram utilizados o óleo essencial de limão siciliano e o óleo essencial de tangerina. Estes óleos essenciais estão entre as composições cítricas (blends) mais vendidas no mundo para indústrias cosméticas, de medicamentos, alimentos e bebidas (BIZZO; MARIA; REZENDE, 2009; BONACCORSI et al., 2011).

A produção de FODs exige a escolha de componentes que favoreçam a formação da película. No desenvolvimento das amostras F1 e F2, a goma xantana e goma carragena foram utilizadas como estabilizantes, espessantes, agentes suspensores e emulsificantes. O sorbato de potássio e benzoato de sódio foram empregados como conservantes. Componentes como acesulfame $\mathrm{k}$, sucralose e manitol foram utilizados como edulcorantes solúveis. A lecitina de soja e polissorbato 80 como emulsionantes, propilenoglicol como umectante e simeticone $30 \%$ como agente antiespuma. polietilenoglicol 400 foi empregado como plastificante e pullulan como polímero formador de filme (ROWE et al., 2009).

É interessante destacar que o pullulan trata-se de um polissacarídeo altamente solúvel em água, com grande capacidade para formar filmes com força mecânica considerável, é biodegradável e comestível (KULKARNI et al., 2010; FERREIRA et al., 2015).

Os FODs propostos neste estudo apresentaram-se flexíveis, com rigidez adequada, ausência de rachaduras e de bolhas, de fácil desintegração em tampão fosfato, $\mathrm{pH}$ variando de 6,5 a 7,5 e propriedades organolépticas agradáveis. 
A Figura 1 demonstra o aspecto dos filmes individuais e sua embalagem original. As preparações apresentaram odor e sabor característicos de limão siciliano (F1) e tangerina (F2). Adicionalmente é importante destacar que o sabor tanto da F1 e F2 se apresentou adocicado. O peso-médio de 20 unidades medindo $3,0 \mathrm{~cm}^{2}$ foi de $0,1156 \pm 0,0030 \mathrm{~g}$ para F1 e $0,1045 \pm 0,0025 \mathrm{~g}$ e tempo de desintegração menor que 30 segundos $\left(37^{\circ} \mathrm{C}\right)$.

No estudo preliminar da estabilidade, não foram observadas alterações nas propriedades dos FODs armazenados em temperatura ambiente e sob exposição à luz natural indireta durante todo tempo de estudo T28 (28 dias). As amostras F1 e F2 foram classificadas com o parâmetro $\mathrm{N}$ (normal, sem alteração visível ou perceptível). Para as amostras armazenadas sob temperatura de $40^{\circ} \pm 2^{\circ} \mathrm{C}$ e $5^{\circ} \pm 2^{\circ} \mathrm{C}$, foram observadas importantes alterações a partir do T7 (sete dias). Tanto a amostra F1 como F2 foram classificadas com o parâmetro MM (muito modificado).

Figura 1. Aspecto dos filmes individuais (a), forma de acondicionamento (b) e embalagem original (c).

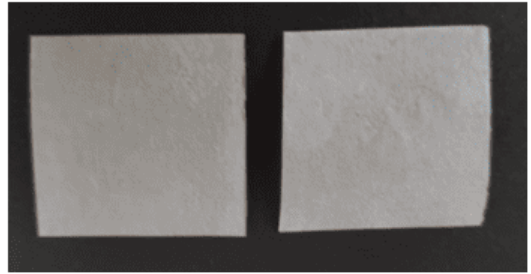

(a)

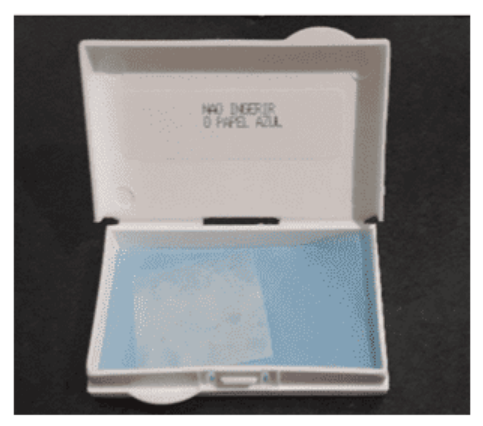

(b)

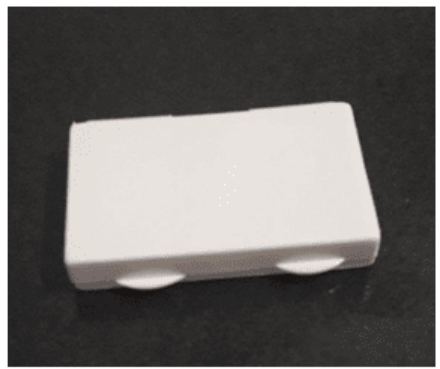

(c)

Fonte: Próprios autores.

Amostras armazenadas em estufa, sob temperatura $40^{\circ} \pm 2^{\circ} \mathrm{C}$, sofreram mudanças no sabor, odor e aspecto. Os filmes adquiriram rigidez, tornaram ressecados, pouco flexíveis e quebradiços (Figura 2) prejudicando também a condução dos demais testes. Amostras armazenadas em geladeira, sob temperatura $5^{\circ} \pm 2^{\circ} \mathrm{C}$, também sofreram mudanças perceptíveis. Nessa condição, os filmes adquiriram aspecto 
gelatinoso, tornaram-se pegajosos inferindo absorção de água. Tais alterações comprometeram as características organolépticas das amostras e outros parâmetros físicos.

Figura 2. Aspecto dos FODs armazenados durante sete dias (T7) sob $40^{\circ} \pm 2^{\circ} \mathrm{C}$ (a) e $5^{\circ} \pm 2^{\circ} \mathrm{C}(\mathrm{b})$.

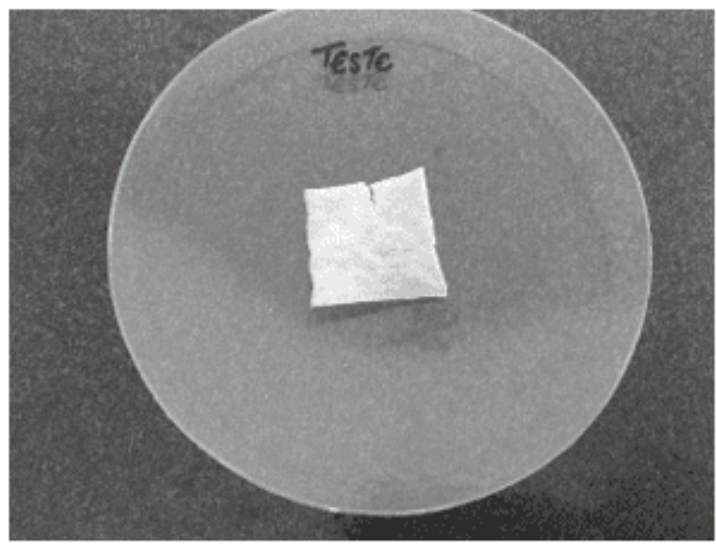

(a)

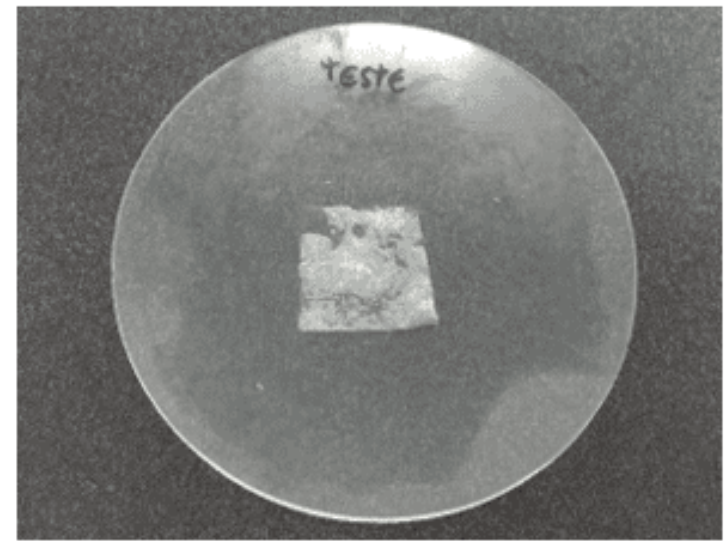

(b)

Fonte: Próprios autores.

Os resultados obtidos demonstram que as condições de armazenamento e o tipo de material de acondicionamento são fatores importantes a serem levados em consideração no desenvolvimento de FODs.

\section{CONCLUSÃO}

Diante dos ensaios realizados e das condições experimentais utilizadas neste estudo foi possível produzir FODs empregando-se óleos essências de limão siciliano e tangerina. As fórmulas propostas e o modo de preparo mostraram-se satisfatórios, as amostras obtidas mantiveram suas características inalteradas por um período de 28 sob temperatura ambiente, entretanto, sofreram alterações quando armazenadas em estufa e geladeira. Os filmes produzidos apresentaram sabor adocicado, odor e sabor muito agradáveis lembrando limão siciliano (F1) e tangerina (F2). Os FODs apresentaram uniformidade e boa aparência, tempo de desintegração reduzido e características organolépticas favoráveis. Embora as amostras sugeridas neste 
estudo se configurem como alternativas promissoras para veiculação de fármacos e liberação de princípios ativos na cavidade bucal, novos estudos devem ser conduzidos para garantir sua produção em larga escala.

\section{REFERÊNCIAS}

ARYA, A. et al. Fast Dissolving Oral Films: An Innovative Drug Delivery System and Dosage Form. Int J ChemTech Res., v. 2, n. 1, p. 576-583, 2010.

BALA, R.; KHANNA, S.; PAWAR, P.; ARORA, S. Orally dissolving strips: A new approach to oral drug delivery system. Int J Pharm Investig, v. 3, n. 2, p. 67, 2013.

BIZZO, H. R.; MARIA, A. C. H.; REZENDE, C. M. Oleos essenciais no Brasil: aspectos gerais, desenvolvimento e perspectivas. Quimica Nova, v. 32, n. 3, p. 588-594, 2009.

BRASIL. Agência Nacional de Vigilância Sanitária. Farmacopeia Brasileira, vol. 1, Brasília: Anvisa, 2019. 546p.

COUTO, R.O. Desenvolvimento de filmes mucoadesivos para liberação de fármacos anestésicos na cavidade bucal. 2015. Tese de Doutorado. Universidade de São Paulo.

DENG, L.; KANG, X.; LIU, Y.; FENG, F.; ZHANG, H. Characterization of gelatin/zein films fabricated by electrospinning vs solvent casting. Food Hydrocolloids, v. 74, p. 324-332, 2018.

FERREIRA, A.O.; BRANDÃO, M. Guia prático da farmácia magistral. Pharmabooks, 2008.

FERREIRA, L.M. et al. Pullulan: an advantageous natural polysaccharide excipient to formulate tablets of alendronate-loaded microparticles. Brazilian Journal of Pharmaceutical Sciences, v. 51, n. 1, p. 27-33, 2015.

GHOSH, T.; GHOSH, A.; PRASAD, D. A review on new generation orodispersible tablets and its future prospective. Int J Pharm Pharm Sci, v. 3, n. 1, p. 1-7, 2011. 
HOFFMANN, E.M.; BREITENBACH, A.; BREITKREUTZ, J. Advances in orodispersible films for drug delivery. Expert opinion on drug delivery, v. 8, n. 3, p. 299-316, 2011.

KESHARI, A.; KUMAR SHARMA, P.; PARVEZ, N. Fast dissolving oral films: an innovative drug delivery system. Structure, v. 20, n. 70, p. 50-500, 2014.

KULKARNI, A. S. et al. Exploration of different polymers for use in the formulation of oral fast dissolving strips. J Curr Pharm Res, v. 2, n. 1, p. 33-35, 2010.

MUSAZZI, U. M. et al. Poly (methyl methacrylate) salt as Im forming material to design orodispersible Ims. Eur J Pharm Sci, v. 115, p. 37-42, 2018.

NAND, P. et al. Mouth Dissolving Tablets- a Novel Drug Delivery System. Intern J Appl Biol Pharm Tech, v. 1, n. 3, 2010.

ORLU, M. et al. Acceptability of orodispersible films for delivery of medicines to infants and preschool children. Drug delivery, v. 24, n. 1, p. 1243-1248, 2017.

PEREIRA, J.F.; MARIM, B.M.; MALI, S. Desenvolvimento de filmes orodispersíveis biopoliméricos à base de amido, goma xantana e gelatina. Iniciação Científica Cesumar, v. 21, n. 1, p. 61-70, 2019.

PERUMAL, V. A. et al. Formulation of monolayered films with drug and polymers of opposing solubilities. International Journal of Pharmaceutics, v. 358, n. 1-2, p. 184191, 2008.

PREIS, M.; PEIN, MI.;BREITKREUTZ, J. Development of a taste-masked orodispersible film containing dimenhydrinate. Pharmaceutics, v. 4, n. 4, p. 551-562, 2012.

RESTA, V.G.; MALI, S. Efeito de sacarose e glicerol como plastificantes em filmes orodispersíveis de amido e gelatina. Iniciação Científica Cesumar, v. 21, n. 1, p. 1525, 2019. 
ROWE, R. C.; SHESKEY, P.; QUINN, M. Handbook of pharmaceutical excipients. Libros Digitales-Pharmaceutical Press, 2009.

RIBEIRO, T.C. et al. Filmes orodispersíveis mucoadesivos baseados em goma gelana e goma de caju para administração via mucosa oral de insulina: avaliação estrutural. Journal of Basic and Applied Pharmaceutical Sciencies, v. 38, Supl. 1, 2017.

SANFELICE, A.M.; TRUITI, M.C. Torrado. Produtos em filme-Inovação na tecnologia de cosméticos. Acta Scientiarum. Health Sciences, v. 32, n. 1, p. 61-66, 2010.

TOMAR, A. et al. Formulation and evaluation of fast dissolving oral film of dicyclomine as potential route of buccal delivery. International Journal of Drug Development \& Research, v. 4, n. 2, p. 408-417, 2012.

Enviado: Outubro, 2019.

Aprovado: Outubro, 2019. 